\title{
Research on Logistic Scheduling Problem with Fuzzy Time Window
}

\author{
Yuqiang Chen ${ }^{1}$, Jianlan Guo ${ }^{2} *$ \\ Department of Computer Engineering DongGuan Polytechnic, Dongguan, \\ Guangdong, China \\ 1,chenyuqiang@126.com,2,rachel0519@126.com
}

\begin{abstract}
Study the problem of logistic scheduling problem with fuzzy time window, construct mathematical model, and propose double objective function method. In the solution process, for the feature of the double objective functions, use the phased. In the first phase, use the chaos PSO to get the optimal solution. In the second phase, use simulated annealing algorithm and the preliminary solutions got from the first phase to solute the objective function.
\end{abstract}

Keywords: logistic scheduling problem; chaos PSO; simulated annealing; fuzzy time window.

\section{Introduction}

Modern logistics is the product of economic globalization and it also is an important service to promote economic globalization. In the recent years, China's logistics industries are also growing rapidly. But our logistics also have problems. Logistics complete the space to space transfer of good with number of power consumption. The consumption is long time and long distance. The logistics scheduling problem with fuzzy time window is an important direction. Researchers from domestic and foreign have done a lot of work. G.B.Alvarenga [1] studied the problem with GA and phased function. Rita Macedo [2] solute the VRP using pseudo-polynomial. Yannis Marinakis [3] studied the hybrid genetic PSO VRP. In domestic, Cheng Jin, Cai Yanguang, Li Yongsheng studied the union scheduling problem. Qu Yuan and Li Min studied the logistic scheduling problem with multiple distribution centers.

This paper is to solute the logistic scheduling problem with fuzzy time window. Studied the improved chaos PSO and simulated annealing problem.

\section{The Mathematical Model of the Logistic Scheduling Problem with Fuzzy Time Window}

In the real life, distribution process is often be disturbed like the customer change the distribution time, car traffic jams, accidents and other unseen circumstances. From the point view of the customer, the distribution is needed to be completed in the prescript time. When the above circumstances occur, customers are able to accept a certain extent of this phenomenon which is the customer service satisfaction problem. The greater the scope beyond the time windows, customer satisfaction will be lower. To the logistics company point of view, they want to find optimal distribution path to save time. But the companies also want good customer satisfaction to company's development. This needs the companies to find the balance point of the cost and the customers' satisfaction. From the above point, this paper use double objective functions to solute the problem. One 
objective function is total cost minimization and the other is service satisfaction optimization.

\subsection{Description of the Problem of Logistic Scheduling Problem with Fuzzy Time Window}

The problem can be described as following: There is a distribution center with I customers. The demand of customers are $q_{i}(i=1,2, \ldots i)$. There are $\mathrm{m}$ distribution cars. There are $\mathrm{k}$ kinds of cars $\varphi_{k}=(1,2 \ldots, k)$ with $\mathrm{k} 1$ represent the 1 car of the $\mathrm{k}$ kind. The max load for each car is $Q_{k}=(1,2 \ldots, m), d_{i j}$ is the distance between customer $\mathrm{i}$ and $\mathrm{j} . T_{i}^{k l}$ is the reaching time of vehicle 1 to customer i. $T_{i}$ is the waiting cost time in serving customer i. $T_{i j}$ is the time from customer $\mathrm{i}$ to customer $\mathrm{j}$. The time windows require the vehicle arrives the customer $\mathrm{i}$ between $\left[A_{i}, B_{i}\right]$. The service satisfaction is $\mathrm{L}, \mathrm{L}$ is between $[0,1]$. If the distribution service is completed in the time window, $\mathrm{L}=1$. The customer has a acceptation scope between $\left[E E T_{i}, E L T_{i}\right]$. There are relationships between different customers.

Then can construct the fuzzy membership function like Eq.1.

$$
L(t)=\left(\begin{array}{lc}
0 & t<E E T \\
f(t)=\frac{t-E E T}{A-E E T} & E E T \leq t<A \\
g(t)=\frac{E L T-t}{E L T-B} & B \leq t<E L T \\
1 & A \leq t<E L T
\end{array}\right.
$$

From the above function, membership function is an trapezoidal function. In the scope of $\left[E E T_{i}, E L T_{i}\right]$, the service satisfaction will change as trapezoidal, it will decrease while time window increase.

\subsection{The Mathematical Model of Problem of Logistic Scheduling Problem with Fuzzy Time Window}

The mathematical model is like following :

Variable definitions :

$$
\begin{aligned}
& x_{i j k l}= \begin{cases}1 & k \text { vehicle I f romi to } j \\
0 & \text { else }\end{cases} \\
& y_{i k l}= \begin{cases}1 & \text { Poi nt i i s compet ed by k vehi cl e I } \\
0 & \text { else }\end{cases}
\end{aligned}
$$

$A_{i}, B_{i}$ is the max and min of time window of customer $\mathrm{i}$.

$E E T_{i}, E L T_{i}$ is the max and min of the fuzzy time window of customer satisfaction

$d_{i j}$ the distance from customer $\mathrm{i}$ to customer $\mathrm{j}$.

$\varphi_{k}=(1,2 \ldots, k)$ car kind set.

$\mathrm{k} 1$ is the 1 car of kink $\mathrm{k}$.

$f_{k}$ is the constant using cost of vehicle of kind $\mathrm{K}$.

$c_{k}$ is the distribution cost of vehicle of kind $\mathrm{K}$.

$\alpha_{i}$ is the service satisfaction.

$S_{i}$ is the reaching time of vehicle to customer i.

$T_{i}$ is the waiting time of serving customer $\mathrm{i}$.

$T_{i j}$ is the cost time in the distribution process from customer $\mathrm{i}$ to customer $\mathrm{j}$.

Objective functions: 


$$
\begin{aligned}
& \min Z=\sum_{k=l}^{K} \sum_{l=1}^{m}\left(f_{k} \sum_{i=1}^{I} x_{0 i k l}\right)+\sum_{i=0}^{I} \sum_{j=0}^{I} \sum_{k=1}^{K} \sum_{l=1}^{m} x_{i j k l} d_{i j} c_{k} \\
& \max L=\sum_{i=1}^{I} L_{i}\left(\sum_{k=1}^{K} \sum_{j=0}^{I} \sum_{i=1}^{m} x_{i j k l} T_{i}^{k l}\right)
\end{aligned}
$$

Constraint functions:

$$
\begin{aligned}
& \sum_{k=1}^{K} \sum_{j=0}^{I} \sum_{i=1}^{m} x_{i j k l}=1 \\
& \sum_{i=0}^{I} q_{i} y_{i k l} \leq Q_{k} \\
& x_{i j k l}\left(T_{i}^{k l}+t_{i} q_{i}+t_{i j}-T_{j}^{k l}\right)=0 \\
& L_{i}\left(\sum_{k=1}^{K} \sum_{j=0}^{I} \sum_{i=1}^{m} x_{i j k l} T_{i}^{k l}\right) \geq \alpha_{i} \\
& S_{i}<S_{j}, T_{l} \in\{(i, j), i, j \in(1,2, \ldots l)\}
\end{aligned}
$$

Eq.2 and Eq. 3 are two objective functions. Eq. 2 is the logistics distribution min $\operatorname{cost} \mathrm{Z}$ which is total of constant cost and distribution cost. Eq.3 is the max function of customer's service satisfaction. Eq.4 used to constrain each customer can only be served once with one vehicle. Eq.5 used to constrain the total distribution weight of good cannot exceed the maximum load.

Eq. 6 is that one vehicle goes to serve customer $\mathrm{j}$ after serving customer $\mathrm{i}$. The relationship meets $T_{i}^{k l}+t_{i} q_{i}+t_{i j}=T_{j}^{k l}$. Eq.7 is used to constrain the customer's service satisfaction of the service which must exceeds $\alpha_{i}$. Eq. 8 is the relationship between customers which customer $\mathrm{i}$ is early to be served than customer $\mathrm{j}$.

\section{Phased Method to Solve Problem of Logistic Scheduling Problem with Fuzzy Time Window}

The model of fuzzy time is complex than the normal model as it has two objective functions. In the real life, logistics companies have time window constraint threshold for maturity customers. Based on a customer delivery tolerance, can only make optimization in the threshold range. Thus can optimize the distribution route, save distribution time, decrease the distribution cost with the consideration of distribution sequence and time which meets the customers' requirement. According to the above reality, we can use two phased solution method to solve the problem.

The two phased method is like Figure 1: 


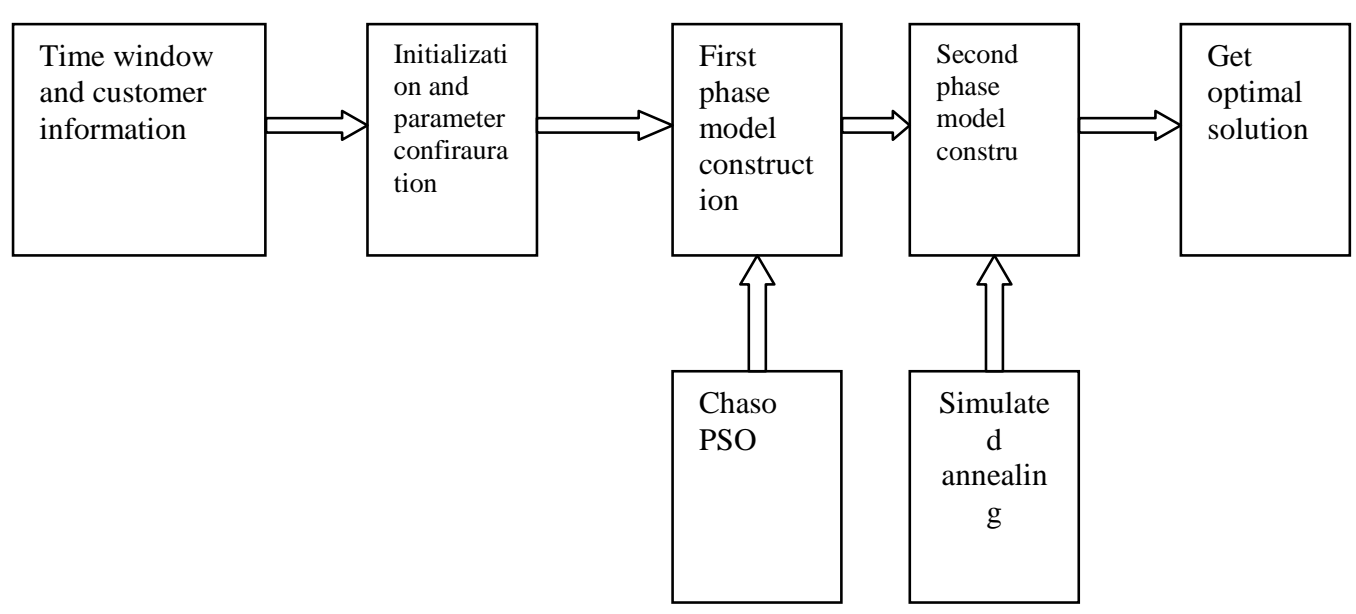

Figure 1. Two Stages Flowchart

The first stage is to solve the problem of getting the lowest cost of the customers whose service satisfaction is bigger than $\alpha_{i}$.

Specific solving process:

Under the condition of customer service satisfaction is bigger than $\alpha_{i}$. As the value of $\alpha_{i}$ is known, then can compute the time window $\left[A_{i}, B_{i}\right]$ through Eq.9.

$$
A=\frac{1}{f_{i}\left(a_{i}\right)} \quad B=\frac{1}{g_{i}\left(a_{i}\right)} \quad i=1,2 \ldots
$$

Then can get the mathematical model as below.

$$
\begin{aligned}
& \min Z=\sum_{k=l}^{K} \sum_{l=1}^{m}\left(f_{k} \sum_{i=1}^{I} x_{0 i k l}\right)+\sum_{i=0}^{I} \sum_{j=0}^{I} \sum_{k=1}^{K} \sum_{l=1}^{m} x_{i j k l} d_{i j} c_{k} \\
& \sum_{k=1}^{K} \sum_{j=0}^{I} \sum_{i=1}^{m} x_{i j k l}=1 \\
& \sum_{i=0}^{I} q_{i} y_{i k l} \leq Q_{k} \\
& x_{i j k l}\left(T_{i}^{k l}+t_{i} q_{i}+t_{i j}-T_{j}^{k l}\right)=0 \\
& A_{i} \leq T_{i}^{k l} \leq B_{i}
\end{aligned}
$$

Eq.11 is the time window constraint under the condition of the customer service satisfaction.

The second stage is making optimization to the preliminary results got from the first stage according to the customer service satisfaction.

Specific solving process:

After the solution of the first stage, we get the preliminary solutions. Basing the preliminary solutions, make distribution time optimization on each distribution route. The goal is to improving customer service satisfaction.

Mathematical model of the second stage:

$$
\max L=\sum_{i=1}^{I} L_{i}\left(\sum_{k=1}^{K} \sum_{j=0}^{I} \sum_{i=1}^{m} x_{i j k l} T_{i}^{k l}\right)
$$




$$
\begin{aligned}
& L_{i}\left(\sum_{k=1}^{K} \sum_{j=0}^{I} \sum_{i=1}^{m} x_{i j k l} T_{i}^{k l}\right) \geq \alpha_{i} \\
& x_{i j k l}\left(T_{i}^{k l}+t_{i} q_{i}+t_{i j}-T_{j}^{k l}\right)=0
\end{aligned}
$$

In the stages, the first stage solving the preliminary solutions is the key. Only save the excellent particles and get preliminary optimal solution in the first stage, that can get more accurate results in the second stage. Due to the two stages solving, in order to solve for more accurate results, this paper uses chaos PSO to optimization and uses simulated annealing in the second stage. The characterize of the simulated annealing is that it less demand the preliminary solutions.

\section{The Solution of the First Stage}

\subsection{Chaos PSO Optimization by Logistic Function}

Logistic function is also called S-function or compression function whose mathematical formula is like bellowing:

$$
y=a+\frac{b}{1+e^{-d x}}
$$

According to the characteristics of nonlinear and everywhere derivable of the Logistic function, this paper uses Eq.19 to make adjustment to the inertia weight factor.

$$
\omega_{i}=\omega_{\max }-\left(\omega_{\max }-\omega_{\min }\right)\left(\frac{2}{1+e^{-d_{i} \frac{N_{i}}{N_{\max }}}}-1\right)
$$

$\omega_{\max }, \omega_{\min }$ is the max and min value of $\omega, N_{\max }$ is the max iteration times, $N_{i}$ is the current iteration time, $\omega_{i}$ is the weight factor to the i particle, parameter $d_{i}$ is computed using the Eq.20.

$$
d_{i}=c \xi_{i}, \xi_{i} \in(0,1), i \in[0, n]
$$

Constant $\mathrm{c}$ is determined according to different circumstance. $\xi_{i}$ is the chaos variable in the ith chaos iteration. $\mathrm{n}$ is the number of populations.

\subsection{The Solution Process of the cChaos PSO Optimized by Logistic Function}

According to the objective function and constraint functions in Eq.9-Eq.14, use chaos PSO optimized by Logistic function to solve the mathematical model in Section.2.

The algorithm process to get preliminary solutions in phase 1 is like bellowing.

Step1 Initialization. Initialize the particle swarm, input basic data to the network. Determine the swarm scope n, learn factor $c_{1} c_{2}$, the max and min value of $\omega \omega_{\max }, \omega_{\min }$, the max chaos iteration times.

Step 2. Compute the inertia weight factor through Eq.18,Eq.19. Determine the customer service satisfaction.

Step 3. Generate particle swarm. Basing the basic parameters to generate the particle swarm with scope $n$.

Step 4. Execute the standard PSO algorithm, save the particle with preliminary good performance. Get $p_{g}$ which is also called $p_{\text {best }}$.

Step 5. Make chaos optimization to the preliminary particles $p_{\text {best }}$ from step 3 to get chaos sequence and make chaos mapping. Find the position to update the optimal solution $p_{g}$.

Step 6. Premature judgment. If the particles fall in premature then let part of the particles with good performance go back step 4 . If not fall in premature then continue the algorithm. There are two features for the premature particles. The first is that the particles 
are extremely gathered. The second is that particle swarms do not change after number of

iterations.

$$
\omega=1.5-\frac{1}{1+r_{1} \exp \left(-r_{2} . \square\right)}
$$

Use Eq.21 to compute the adaptive variance of $\sigma^{2}$ of the particle swarm. Make comparison with the predefined minimum population adaptive variance $\sigma_{\min }^{2}$. If $\sigma^{2} \leq \sigma_{\min }^{2}$, then the particle is extremely gathered and fall in premature.

Step 7. Algorithm stop condition. If the iteration times to get the optimal solution $p_{g}^{\prime}$ is bigger than the predefined max iteration time, then the algorithm stops.

Step 8. Get the global optimal solution which is the optimal distribution route.

Step 9. Algorithm stops.

\section{Solve the Second Phase using Simulated Annealing Algorithm}

\subsection{The Mechanism of the Simulated Annealing Algorithm}

Basing on the theory of the simulated annealing algorithm, the probability of solid trending to balance at a temperature $\mathrm{T}$ is $e-\Delta E / k T . \Delta E$ is the solid internal energy change, $\mathrm{K}$ is constant. The simulated annealing algorithm firstly find the initial solution and control parameter $\mathrm{t}$. Then get the update solution, then compute the objective function minus, then update solution or abandon the solution. Loop the above sequence to the end of the algorithm to find the optimal solution. The control parameter will decrease with the increase of the iteration.

The biggest feature of simulated annealing algorithm is less dependent on the initial solutions. This provides convenient to the solution in the second stage.

The procedures of simulated annealing algorithm[6] :

(1) Firstly get the basic parameters like the initial solution ${ }^{x_{o}}$, initial temperature, current temperature, max iteration time, etc.

(2) If the current temperature reaches the inside cycle temperature, then go to (3), else, do field operations, select a temporary solution $x_{j}$, compute $\Delta E_{i j}=E\left(x_{j}\right)-E\left(x_{i}\right)$, if $\Delta E_{i j}$ is less or equal 0 or $\exp \left(-\Delta E_{i j} / t\right)>\operatorname{rand}(0,1)$, then let $x_{i}=x_{j}$, repeat step (2).

(3) Update the iteration time $\mathrm{k}=\mathrm{k}+1$, according the temperature control function to update the current temperature. The temperature control function is $y\left(t_{k}\right)=t_{k+1}$. If meets the algorithm stop condition, then go to step (4), else go back to step (2).

(4) Output results, algorithm ends.

\subsection{Algorithm Process}

According to the mathematical model, use the optimal solutions got from the first stage as the initial solutions. The second stage of the simulated annealing algorithm is like bellowing.

Firstly input the external parameters like car model, customer information, etc. Then initialize the objective function. Use the preliminary optimal solution $\mathrm{O}$, control parameter t. Conduct field operations, generate a neighboring solution $\mathrm{O}^{\prime}$, compute the energy difference $\Delta E_{i j}=E\left(x_{j}\right)-E\left(x_{i}\right)$, if $\Delta E_{i j}$ equals or less than 0 or $\exp \left(-\Delta E_{i j} / t\right)>\operatorname{rand}(0,1)$, then let $\mathrm{O}=\mathrm{O}^{\prime}$. Generate the new solution from the preliminary solution, compute the objective functions difference, update or abandon the new solution. Repeat the above process with the decreasing of control parameter t. Otherwise return the field operation steps. Update the iterations $\mathrm{k}=\mathrm{k}+1$, update the current temperature with 
reference to the temperature control function which is like $y\left(t_{k}\right)=t_{k+1}$. If meet the algorithm end condition, the algorithm terminates and get the optimal solution.

There are two criteria to determine algorithm convergence. The first is max iteration times. The second is when the temperature is lower than a certain value or temperature does not change several times.

$$
\exp \left(-\Delta E_{i j} / t\right)>\operatorname{rand}(0,1)
$$

\section{Experiment Results and Analysis}

The experiments are done on a pc with Intel(R) Core $2 \mathrm{CPU} 2.66 \mathrm{GHz}$, memory 2.0G, Matlab7. The specific content is like following.

A logistic company has a distribution center. The company has four kinds of cars and 8 customers. Suppose the upper and low limit of the time window and the customer acceptable fuzzy time window is known. The customer 1 is required to be served before customer 3. The experiments are to determine the car type, distribution routes and distribution time to minimize the total cost. The details are shown as below.

Table 1. Vehicle Information

\begin{tabular}{|c|c|c|c|c|}
\hline Car type & Number & Load & Constant cost & Driving cost \\
\hline A & 3 & 1100 & 100 & 1.2 \\
\hline B & 1 & 1130 & 110 & 1.5 \\
\hline C & 4 & 2000 & 95 & 1.1 \\
\hline D & 2 & 850 & 100 & 1.6 \\
\hline E & 2 & 990 & 80 & 1.3 \\
\hline F & 4 & 900 & 120 & 1.4 \\
\hline
\end{tabular}

Table 2. Customer Distance

\begin{tabular}{|c|c|c|c|c|c|c|c|c|c|}
\hline Distance & 0 & 1 & 2 & 3 & 4 & 5 & 6 & 7 & 8 \\
\hline 0 & 0 & 10 & 9 & 30 & 21 & 23 & 34 & 40 & 34 \\
\hline 1 & 10 & 0 & 36 & 10 & 30 & 20 & 18 & 14 & 41 \\
\hline 2 & 9 & 36 & 0 & 12 & 21 & 19 & 9 & 22 & 9 \\
\hline 3 & 30 & 10 & 12 & 0 & 35 & 37 & 41 & 25 & 28 \\
\hline 4 & 21 & 30 & 21 & 35 & 0 & 20 & 32 & 14 & 12 \\
\hline 5 & 23 & 20 & 19 & 37 & 20 & 0 & 18 & 8 & 25 \\
\hline 6 & 34 & 18 & 9 & 41 & 32 & 18 & 0 & 17 & 7 \\
\hline 7 & 40 & 14 & 22 & 25 & 14 & 8 & 17 & 0 & 32 \\
\hline 8 & 34 & 41 & 9 & 28 & 12 & 25 & 7 & 32 & 0 \\
\hline
\end{tabular}

Customers demand and time windows are shown in table 3. In table 3, A is the upper limit of the time window, B is the low limit of the time window, EET is the upper limit of the customer acceptable fuzzy time window, ELT is the low limit of the customer acceptable fuzzy time window. In order to facilitate the calculation of the model, this paper set the maximum acceptable time window EET is A with reduction of 10 and the minimum acceptable time window ELT is B with reduction of 10 .

Table 3. Customer Demand and the Time Window Information

\begin{tabular}{|c|c|c|c|c|c|}
\hline Customer & Demand & A & B & EET & ELT \\
\hline 0 & 0 & 0 & 200 & 0 & 210 \\
\hline 1 & 400 & 23 & 53 & 13 & 63 \\
\hline 2 & 280 & 19 & 39 & 9 & 49 \\
\hline
\end{tabular}




\begin{tabular}{|c|c|c|c|c|c|}
\hline 3 & 250 & 20 & 56 & 10 & 66 \\
\hline 4 & 450 & 43 & 86 & 33 & 96 \\
\hline 5 & 200 & 62 & 97 & 52 & 107 \\
\hline 6 & 500 & 56 & 90 & 46 & 100 \\
\hline 7 & 300 & 81 & 103 & 71 & 113 \\
\hline 8 & 300 & 75 & 99 & 65 & 109 \\
\hline
\end{tabular}

Experiment results:

Use the algorithm proposed in this paper to do simulated analysis of the above case. In order to reflect customer satisfaction's impact on results, we use constant customer satisfaction $\alpha_{i}$.We analyze the circumstance when $\alpha_{i}$ equals 1 , not less than 0.9 and not less than 0.8. The experiment results are listed in table 4-6.

Table 4. The Experimental Results when ${ }^{\alpha_{i}}=1$

\begin{tabular}{|c|c|c|}
\hline Distribution & \multicolumn{2}{|c|}{ Car type } \\
\hline Route 1 & C & $0-4-6-7-0$ \\
\hline Route 2 & C & $0-1-5-0$ \\
\hline Route 3 & A & $0-8-3-2-0$ \\
\hline Distribution cost & \multicolumn{2}{|c|}{386.5} \\
\hline
\end{tabular}

Table 5. The Experimental Results when $\alpha_{i} \geqq 0.9$

\begin{tabular}{|c|c|c|}
\hline Distribution & \multicolumn{2}{|c|}{ Car type } \\
\hline Route 1 & C & $0-2-4-8-0$ \\
\hline Route 2 & C & $0-7-5-0$ \\
\hline Route 3 & A & $0-1-3-0$ \\
\hline Route 5 & E & $0-6-0$ \\
\hline Distribution cost & \multicolumn{2}{|c|}{366.4} \\
\hline
\end{tabular}

Table 6. The Experimental Results when ${ }^{\alpha_{i}} \geqq 0.8$

\begin{tabular}{|c|c|c|}
\hline Distribution & \multicolumn{2}{|c|}{ Car type } \\
\hline Route 1 & C & $0-3-8-0$ \\
\hline Route 2 & C & $0-7-5-2-0$ \\
\hline Route 4 & A & $0-4-1-0$ \\
\hline Route 4 & E & $0-6-0$ \\
\hline Distribution cost & \multicolumn{2}{|c|}{344.8} \\
\hline
\end{tabular}

From the above experiments data we can conclude, when $\alpha_{i}=1$, the model is same as the hard time window problem. When $\alpha_{i} \geqq 0.9$, the distribution routes change and total distribution cost decrease. When $\alpha_{i} \geqq 0.8$ also can promise completing distribution and 
the distribution cost decrease reference to the above two circumstances. So, control the customer satisfaction in a reasonable range can obviously decrease the distribution cost of the logistics company.

\title{
7. Summary
}

The paper introduces the solution method of the logistics scheduling problem with fuzzy time window. Construct mathematical model, introduce customer satisfaction as the second objective function. Use two methods to solve the mathematical model. In the first stage, use chaos PSO algorithm. In the second stage, use the simulated annealing algorithm. Finally, do simulation analysis which shows that it is reasonable to use two methods to solve the problem. The conclusion is that control the customer satisfaction in a reasonable range can obviously decrease the distribution cost of the logistics company.

\section{Acknowledgements}

The work described in this paper was supported by National Natural Science Foundation of China (No.61106019), also supported by Guangdong Provincial of Science and Technology Foundation (No. 2012B040500007), also supported by the excellent young teachers program project of Guangdong Province(No:Yq2013201), and also supported by the project of Chinese Institute of logistics (2014CSLKT3-205).

\section{References}

[1] Y. Marinakis and M. Marinaki, "Expert System with Applications", vol. 37, (2010), pp.1446-1455.

[2] G. B. Alvarenga, G. R. Mateus and G. de Tomi, "Computers \& Operations Research, vol. 34, (2007), pp. 1561-1584.

[3] R. Macedo, C. Alves, J. M. Valerio de Carvaiho, F. Clautiaux and S. Hanafi, "European Journal of Operational Research, vol. 214, (2011), pp.536-545.

[4] Z. Yi, L. H. Long and W. Rui, Journal of Jilin University, Science Edition, vol. 2, (2010), pp.197-203.

[5] C. Y. Guang and S. Kai, "Computer Integrated Manufacturing Systems", vol. 12, no. 11, (2006), pp. 8487.

[6] Z. Yi, L. H. Long and W. Rui, "Journal of Jilin University”, Science Edition, vol. 2, (2010), pp. 197-203.

[7] S. Suresh, P. B. Sujit and A. K. Rao, "Composite Structures", vol. 81, no. 4, (2007), pp.598-605.

[8] A. Gohary and A. S. Ruzaiza, "Chaos, Solitons and Fractals", vol. 34, no. 2, (2007), pp.443-453.

[9] G. W. Jeon, H. R. Leep and J. Y. Shim, "Computers \& Industrial Engineering, vol. 53, (2007), pp. 680692.

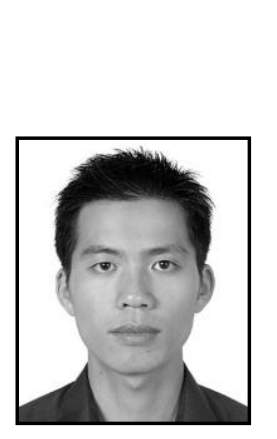

\begin{abstract}
Authors
Yuqiang Chen. Chen Yuqiang was born in 1980, graduated from Guangdong University of Technology, and is associate professor in Dongguan polytechnical College. His present research interests include automatic control and computer applications.
\end{abstract}

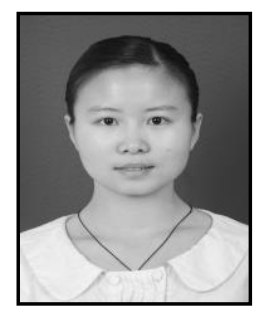

Jianlan Guo. Guo Jianlan was born in 1984, graduated from Guangdong University of Technology, and is senior engineer in Dongguan polytechnical College. Her present research interests include automatic control and computer applications. 
International Journal of Grid Distribution Computing Vol. 8, No. 1 (2015) 